\title{
Article \\ Prognostic Role of Androgen Receptor Expression in HER2+ Breast Carcinoma Subtypes
}

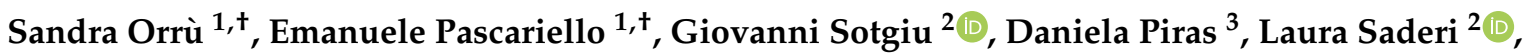 \\ Maria Rosaria Muroni ${ }^{2}$, Ciriaco Carru ${ }^{4}\left(\mathbb{D}\right.$, Caterina Arru ${ }^{4}$, Cristina Mocci ${ }^{1}$, Giampietro Pinna ${ }^{1}$, \\ Raffaele Barbara ${ }^{5}$, Paolo Cossu-Rocca ${ }^{2,6}$ and Maria Rosaria De Miglio ${ }^{2, *}$ (1)
}

check for

updates

Citation: Orrù, S.; Pascariello, E.;

Sotgiu, G.; Piras, D.; Saderi, L.;

Muroni, M.R.; Carru, C.; Arru, C.;

Mocci, C.; Pinna, G.; et al. Prognostic

Role of Androgen Receptor

Expression in HER2+ Breast

Carcinoma Subtypes. Biomedicines

2022, 10, 164. https://doi.org/

10.3390/biomedicines10010164

Academic Editors: Shin-Cheh Chen and Chun-Yu Liu

Received: 6 December 2021

Accepted: 11 January 2022

Published: 13 January 2022

Publisher's Note: MDPI stays neutral with regard to jurisdictional claims in published maps and institutional affiliations.

Copyright: (C) 2022 by the authors. Licensee MDPI, Basel, Switzerland. This article is an open access article distributed under the terms and conditions of the Creative Commons Attribution (CC BY) license (https:// creativecommons.org/licenses/by/ $4.0 /)$.
1 Department of Pathology, "A. Businco" Oncologic Hospital, ARNAS Brotzu, Via Edward Jenner 1, 09121 Cagliari, Italy; sandra.orru@aob.it (S.O.); anatomiapatologica.businco@aob.it (E.P.); cristina.mocci@aob.it (C.M.); g.pinna33@studenti.unica.it (G.P.)

2 Department of Medical, Surgical and Experimental Sciences, University of Sassari, Via P. Manzella 4 , 07100 Sassari, Italy; gsotgiu@uniss.it (G.S.); 1saderi@uniss.it (L.S.); mrmuroni@uniss.it (M.R.M.); rocco@uniss.it (P.C.-R.)

3 Struttura Complessa Epidemiologia e Registro Tumori Nord Sardegna, ATS Sardegna, Via Rizzeddu 2, 07100 Sassari, Italy; dani.piras@atssardegna.it

4 Department of Biomedical Sciences, University of Sassari, Viale San Pietro 43, 07100 Sassari, Italy; carru@uniss.it (C.C.); 30039590@studenti.uniss.it (C.A.)

5 Department of Radiotherapy, “A. Businco" Oncologic Hospital, ARNAS Brotzu, Via Edward Jenner 1, 09121 Cagliari, Italy; raffaele.barbara@aob.it

6 Department of Diagnostic Services, "Giovanni Paolo II" Hospital, ASSL Olbia-ATS Sardegna, Via Bazzoni-Sircana, 07026 Olbia, Italy

* Correspondence: demiglio@uniss.it; Tel.: +39-079228016

+ These authors contributed equally to this work.

Abstract: HER2+ breast cancer (BC) is an aggressive subtype representing a genetically and biologically heterogeneous group of tumors resulting in variable prognosis and treatment response to HER2-targeted therapies according to estrogen (ER) and progesterone receptor (PR) expression. The relationship with androgen receptors (AR), a member of the steroid hormone's family, is unwell known in BC. The present study aims to evaluate the prognostic impact of AR expression in HER2+ BC subtypes. A total of $695 \mathrm{BC}$ s were selected and reviewed, AR, ER, PR and HER2 expression in tumor cells were examined by immunohistochemical method, and the SISH method was used in case of HER2 with equivocal immunohistochemical score (2+). A high prevalence of AR expression $(91.5 \%)$ in BC HER+ was observed, with minimal differences between luminal and non-luminal tumor. According to steroid receptor expression, tumors were classified in four subgroups, including BC luminal and non-luminal HER2+ expressing or not AR. The luminal BC HER2 + AR+ was associated with lower histological grade, lower tumor size, higher PR expression and lower HER2 intensity of expression (2+). Also, the non-luminal tumors AR+ showed lower tumor size and lower prognostic stage but frequently higher grade and higher HER2 intensity of expression (3+). These findings should suggest a different progression of luminal and non-luminal tumors, both expressing AR, and allow us to speculate that the molecular mechanisms of $\mathrm{AR}$, involved in the biology of $\mathrm{BC}$ HER2 + AR+, differ in relation to ER and PR expression. Moreover, AR expression may be a useful predictor of prognosis for overall survival (OS) in HER2+ BC subtypes. Our findings suggest that AR expression evaluation in clinical practice could be utilized in clinical oncology to establish different aggressiveness in BC HER2+ subtypes.

Keywords: HER2 expression; AR expression; breast cancer; prognosis; immunohistochemistry

\section{Introduction}

Breast cancer $(\mathrm{BC})$ is a heterogeneous disease enclosing several entities with different morphologic, prognostic and therapeutic features [1]. Invasive breast cancer is classified 
according to histology and immunohistochemistry (i.e., ER, PR, HER2 overexpression, and/or HER2 gene amplification, and Ki67 proliferation index) [1,2]. Surrogate molecular classification of BC by means of immunohistochemistry defines specific subtypes, such as Luminal A (ER and PR positive, HER2 negative, low Ki67), Luminal B (ER positive, PR positive/negative, HER2 negative, high Ki67), Luminal B HER2+ (ER positive, PR positive/negative, HER2 positive, any Ki67), HER2+ non-luminal (ER-PR negative, HER2 positive, high Ki67), and triple negative (ER-PR-HER2 negative, high Ki67) [3,4]. BC immunohistochemistry surrogate classification has an actual utility in the management of BC patients having significant prognostic and predictive value [4].

HER2+ BCs include different histological subtypes: invasive BC no special type (IBCNST), lobular, micropapillary, apocrine, rarely mucinous [5]. Immunophenotypically, HER2+ BCs variably express hormone receptors.

HER2 protein overexpression or gene amplification, which accounts for $~ 15-18 \%$ of all BC, is frequently associated with high invasiveness and worse prognosis without appropriate therapy [6]. HER2 signaling activates PI3K/AKT and RAS/RAF/MAPK pathways, favoring cell proliferation, growth, invasion, and angiogenesis [7]. Sequential chemotherapy combined with anti-HER2 therapy is the landmark for HER2+ BC treatment, both in the neoadjuvant, adjuvant and metastatic setting [8]. Five drugs were made available by the FDA-U.S. for HER2+ BCs treatment: trastuzumab, lapatinib, neratinib, TDM-1, and pertuzumab [9]. Not all HER2+ BC patients clinically benefit from trastuzumab treatment owing to intrinsic or acquired resistance [10], which depends on persistence of HER2 activation, associated with other EGFR members compensatory activations, or structural aberrations of HER2 protein altering trastuzumab binding, activation/inactivation of members of PI3K/AKT downstream to HER2, or activation of different tyrosine-kinases receptors [11-15].

The androgen receptor is a member of the steroid nuclear receptor superfamily, and it is widely expressed in different subtypes of breast cancer, including HER2+ tumors. It could be a promising prognostic and predictive factor and therapeutic target in BC [16-19]. AR expression and its biologic effects can vary, depending on ER expression [20-22]: 90\% of ER-positive BCs express AR, which inhibits ER activity and improves prognosis, though it can induce resistance to tamoxifen and/or aromatase inhibitors, which can be reverted by AR inhibitors [23-26]. AR was found in $~ 20 \%$ of Triple Negative BCs, with controversial findings on its prognostic role $[27,28]$. Most HER2+ BCs overexpress AR [20,29], but its prognostic role is unclear $[30,31]$. Several studies showed that AR can promote the growth of HER2 + BC cells by cross-talking with the HER2 signaling. Ni et al. found that AR activated by androgen/WNT7B binds to FOXA1 and $\beta$-catenin and triggers HER 2 and HER3 downstream, promoting the proliferation [32]. Lin et al. showed that co-expression of HER2, ER, and AR reduces tumor invasiveness, improving patient outcomes [33]. The expression of AR in HER2+, ER-negative BC patients has been associated with worse clinical outcomes [23]. The AR receptor blockade could represent a therapeutic option in HER2+ BC. On the other hand, HER2+ BC patients treated with first-line trastuzumab show better disease-free survival (DFS) and overall survival (OS) in case of high AR expression [34].

The aim of the present study was to evaluate AR expression in a large series of HER2+ BC, to establish its prognostic role and clinical-pathological relationship.

\section{Materials and Methods}

A retrospective cohort of 695 BC patients, diagnosed between 2012 and 2021, was recruited through a complete review of surgical samples and medical records from the archives of the Department of Histopathology of Oncologic Hospital in Cagliari, Italy. The inclusion criteria were histologically invasive BC, HER2 positive expression determined by immunohistochemistry (IHC) and/or and in situ hybridization (SISH) assay, availability of formalin-fixed, paraffin embedded (FFPE) tumor blocks and clinico-pathological data. The exclusion criteria were in situ BC and HER2 negative status. Three $\mu$ m-thick 
tissue sections of FFPE specimens were cut for hematoxylin and eosin staining (H\&E), immunohistochemistry, and SISH analysis.

The study protocol was approved by the local research ethics committee (File number PG/2021/14264); and followed the Italian law on guidelines for the implementation of retrospective observational studies (G.U. n. 76, 31 March 2008). Only coded data were collected in order to protect patient confidentiality.

\subsection{Immunohistochemistry}

The immunohistochemistry analysis was performed using specific antibodies against ER, Clone SP1 (Ventana Medical Systems Inc., Tucson, AZ, USA); PR Clone 1E2 (Ventana Medical Systems); Ki67, Clone 30-9 (Roche Diagnostics K.K., Tokyo, Japan); AR Clone SP107 (Cell-MarqueTM, Rocklin, CA, USA); HER2 PATHWAY Clone 4B5 (Ventana Medical Systems). Immunostaining was performed using the Ventana Benchmark XT staining system with Optiview DAB detection kit. In cases of HER2 with equivocal immunohistochemical score (2+), we performed HER2 gene amplification by ultra-View SISH Detection Kit (Ventana Medical Systems). Evaluation of immunostaining and SISH for HER2 was based on American Society of Clinical Oncology /College of American Pathologists (ASCO/CAP) recommendations [6]. ER, PR and AR expression was considered positive if at least 10\% immunostained tumor nuclei were detected in the sample [17]. Ki67 was scored low if $<14 \%$ of tumor nuclei were positive and high if $\geq 14 \%$ of tumor nuclei were positive [35].

\subsection{Baseline Data}

Demographic and clinico-pathological information were extracted from medical records, as well as age at diagnosis, histologic type, histologic grade, tumor site, and TNM classification. Histologic tumor type was established according to the UICC-WHO criteria [5]. Histologic tumor grade was determined in accordance with the Nottingham guideline [36]. TNM staging was described in accordance with the 8th edition of the American Joint Committee on Cancer criteria (AJCC) [37]. Lymph node ratio was described as the ratio between the positive lymph nodes number and the evaluated lymph nodes number. The cut-off points of lymph node ratio were: $<0.21,0.21-0.65$, and $>0.65$ [38]. The time between the date of $\mathrm{BC}$ diagnosis and the date of death define the OS.

\subsection{Statistical Analysis}

Quantitative variables were described by median and interquartile range (IQR) according to non-normal distribution of variables, whereas absolute and relative (percentages) frequencies were used for qualitative variables. Statistical differences for qualitative variables were evaluated using Chi2 or Fisher's exact tests, when appropriate. Logistic regression analysis was performed to evaluate the association between mortality, IHC BC subtypes, clinico-pathological features, and molecular variables. A Kaplan-Meier curve and Log-Rank test were performed to describe OS according to IHC BC subtypes, clinicopathological and molecular variables. The statistical significance was set up at $p<0.05$. Statistical analysis was carried out using STATA ${ }^{\circledR} 16$ (StataCorp, College Station, TX, USA).

\section{Results}

A total of 695 HER2+ BC patients were selected. Tumors were grouped into four subtypes based on hormone receptors (ER, PR and AR): Luminal B (LB) HER2 + AR+ (ER $\geq 10 \%, \mathrm{PR} \geq 10 \%$ or $<10 \%$, and $\mathrm{AR} \geq 10 \%)$; Luminal B HER $2+\mathrm{AR}-(\mathrm{ER} \geq 10 \%$, $\mathrm{PR} \geq 10 \%$ or $<10 \%$, and $\mathrm{AR}<10 \%)$; HER $2+\mathrm{AR}+(\mathrm{ER}<10 \%, \mathrm{PR}<10 \%$ and $\mathrm{AR} \geq 10 \%)$; HER $2+\mathrm{AR}-(\mathrm{ER}<10 \%, \mathrm{PR}<10 \%$, and AR $<10 \%)$. Table 1 shows subtypes proportion based on the state of the hormonal receptors and the results of HER2 intensity immunostaining for each subtype. Figure 1 shows representative IHC positive results obtained for AR and HER2, and SISH positive results for HER2 with equivocal immunostaining intensity (2+). All tumors with HER2 IHC 2+ included in the study were SISH positive. 
Table 1. HER2-positive BC subtypes proportion and immunohistochemistry positivity frequency.

\begin{tabular}{ccccc}
\hline \multirow{2}{*}{ IHC BC Subtypes } & Number & $\mathbf{\%}$ & \multicolumn{2}{c}{ HER2 IHC Intensity } \\
\cline { 4 - 5 } & & & $\mathbf{2 +}$ & $\mathbf{3 +}$ \\
\hline Luminal B HER2 + AR+ & 396 & 57.0 & 126 & 270 \\
\hline Luminal B HER2 + AR- & 18 & 2.6 & 6 & 12 \\
\hline HER2 + AR+ & 241 & 34.7 & 22 & 219 \\
\hline HER2 + AR - & 40 & 5.7 & 5 & 35
\end{tabular}

IHC: Immunohistochemistry.
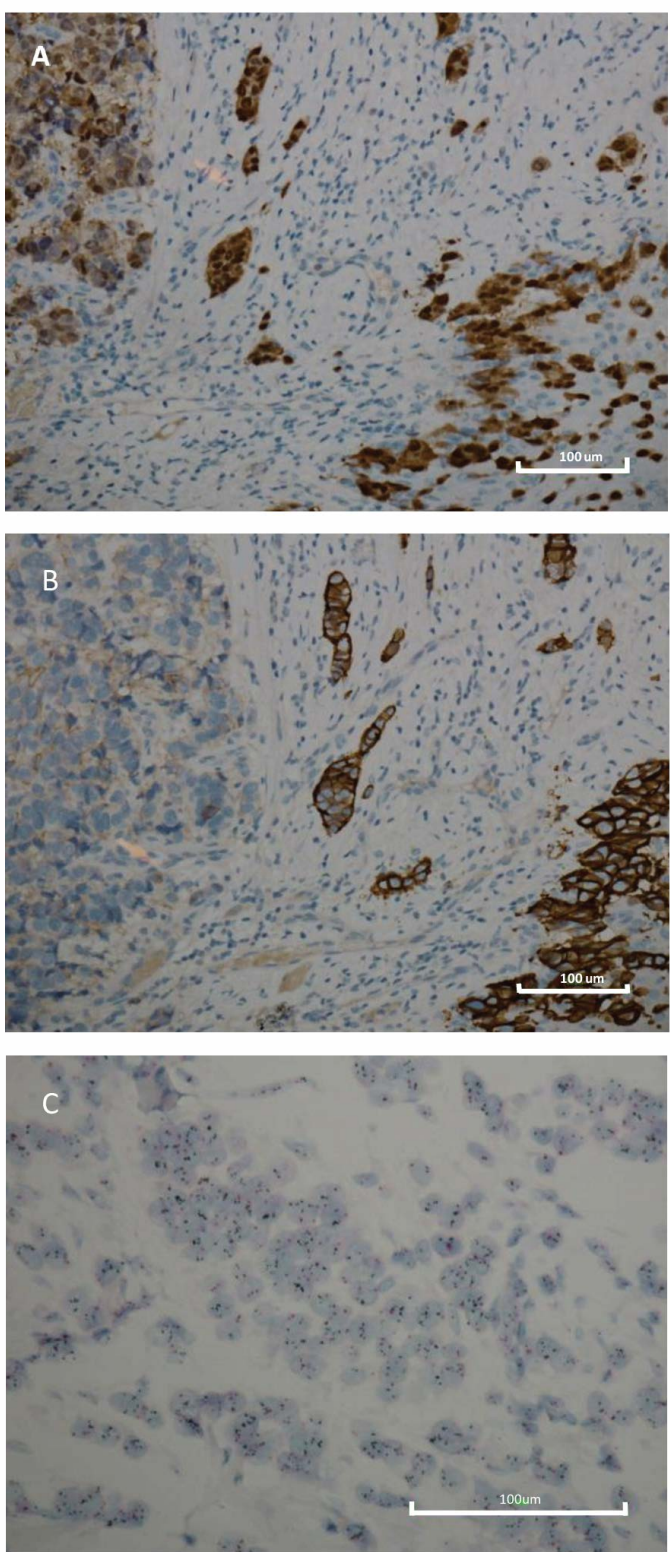

Figure 1. Immunohistochemistry and silver in situ hybridization features of breast cancer. (A) Immunohistochemistry for AR displaying diffuse and intense nuclear immunoreactivity (original magnification 200×); (B) Immunohistochemistry for HER2 displaying diffuse and intense membranous immunoreactivity (original magnification $200 \times$ ); (C) Determination of HER2 gene status using the Dual SISH kit (Ventana) of a breast carcinoma with HER2 gene amplification; HER2 (black) and Chr17 (red). Scale bar: $100 \mu \mathrm{m}$. 
The median (range) age at diagnosis was 58 (27-91) years, with 363 (52.2\%) equal to or older than 58 years. $590(85.0 \%)$ tumors were ductal, 30 (4.3\%) lobular, and $39(5.6 \%)$ apocrine; less common were other histologic types, such as mixed (ductal + lobular, 1.9\%), micropapillary/papillary (2.2\%), and mucinous (1.0\%). Pathological tumor staging was pT1 in $203(32.6 \%)$ cases, pT2 in 288 cases $(46.2 \%)$; pT3 in 27 cases $(4.3 \%)$; and pT4 in 52 cases (8.4\%). Pathological lymph node status was distributed as follows: pN0 in 321 (55.9\%); pN1 in 134 (23.3\%); pN2 in 73 (12.7\%); pN3 in 46 (8.0\%). A total of 54 (7.8\%) were metastatic; $83.7 \%$ of HER2-positive BC were G3. Ninety nine out of 695 patients (14.2\%) died (Table 2 ).

AR positivity was detected in $91.5 \%$ of all tumors, specifically in $95.2 \%$ of luminal and in $84.2 \%$ of non-luminal subtypes.

The median age at diagnosis of HER2 $+\mathrm{AR}+\mathrm{BC}$ patients were 59 years $(p: 0.045)$ and showed the following features: histological grade 2 was found in $17.3 \%$ of AR+ tumors vs. $5.2 \%$ of AR - tumors ( $p$ : 0.020), pT1 in 33.7\% of AR+tumors vs. $20.8 \%$ of AR- ones ( $p$ : 0.020$)$, prognostic stage I in $42.8 \%$ AR+ BC vs. $16.3 \%$ of those with AR negativity ( $p$ : 0.001). Moreover, HER2+ AR+ BCs were more frequently associated with ER and PR expression $\geq 10 \%$ ( $p<0.0001$ and $p<0.001$, respectively). There were no significant differences between site, histological type, lymph node status and ratio, metastasis, ki67 and HER2 expression (Table 3).

Table 2. Clinico-pathological features of HER2-positive breast cancer.

\begin{tabular}{|c|c|c|}
\hline \multicolumn{2}{|l|}{ Variables } & $n=695$ \\
\hline \multicolumn{2}{|c|}{ Median (IQR) age, years } & $58(49-68)$ \\
\hline \multirow{2}{*}{ Age (year), $n(\%)$} & $<58$ & $332(47.8)$ \\
\hline & $\geq 58$ & $363(52.2)$ \\
\hline \multirow{2}{*}{ Site, $n(\%)$} & Left & $396(57.2)$ \\
\hline & Right & $296(42.8)$ \\
\hline \multirow{6}{*}{ Histologic type, $n(\%)$} & Ductal & $590(85.0)$ \\
\hline & Apocrine & $39(5.6)$ \\
\hline & Lobular & $30(4.3)$ \\
\hline & Micropapillary/papillary & $15(2.2)$ \\
\hline & Mixed (ductal + lobular) & $13(1.9)$ \\
\hline & Mucinous & $7(1.0)$ \\
\hline \multirow{2}{*}{ Histologic grade, $n(\%)$} & G2 & $112(16.3)$ \\
\hline & G3 & $575(83.7)$ \\
\hline \multirow{5}{*}{ Pathological tumor size, $n(\%)$} & $\mathrm{pT0} *$ & $53(8.5)$ \\
\hline & pT1 & $203(32.6)$ \\
\hline & pT2 & $288(46.2)$ \\
\hline & pT3 & $27(4.3)$ \\
\hline & pT4 & $52(8.4)$ \\
\hline \multirow{4}{*}{ Pathological lymph node status, $n(\%)$} & pN0 & $321(55.9)$ \\
\hline & $\mathrm{pN} 1$ & $134(23.3)$ \\
\hline & $\mathrm{pN} 2$ & $73(12.7)$ \\
\hline & $\mathrm{pN} 3$ & $46(8.0)$ \\
\hline \multirow{3}{*}{ Lymph node ratio, $n(\%)$} & $\leq 0.20$ & $400(72.3)$ \\
\hline & $0.21-0.65$ & $106(19.2)$ \\
\hline & $>0.65$ & $47(8.5)$ \\
\hline
\end{tabular}


Table 2. Cont.

\begin{tabular}{|c|c|c|}
\hline \multicolumn{2}{|c|}{ Variables } & $n=695$ \\
\hline \multirow{4}{*}{ Prognostic stage, $n(\%)$} & I & $249(40.8)$ \\
\hline & II & $174(28.5)$ \\
\hline & III & $134(21.9)$ \\
\hline & IV & $54(8.8)$ \\
\hline \multicolumn{2}{|c|}{ Metastasis, $n(\%)$} & $54(7.8)$ \\
\hline \multirow{2}{*}{ Proliferation index (Ki-67), $n(\%)$} & $<14 \%$ & $9(1.3)$ \\
\hline & $\geq 14 \%$ & $686(98.7)$ \\
\hline \multirow{2}{*}{ PR expression, $n(\%)$} & $<10 \%$ & $455(65.5)$ \\
\hline & $\geq 10 \%$ & $240(34.5)$ \\
\hline \multirow{2}{*}{ ER expression, $n(\%)$} & $<10 \%$ & $281(40.4)$ \\
\hline & $\geq 10 \%$ & $414(59.6)$ \\
\hline \multirow{2}{*}{ AR expression, $n(\%)$} & $<10 \%$ & $59(8.5)$ \\
\hline & $\geq 10 \%$ & $636(91.5)$ \\
\hline \multirow[t]{2}{*}{ HER2 expression, $n(\%)$} & $2+$ & $159(22.9)$ \\
\hline & $3+$ & $536(77.1)$ \\
\hline \multicolumn{2}{|c|}{ Mortality, $n(\%)$} & $99(14.2)$ \\
\hline
\end{tabular}

IQR: Interquartile range, $n$ : Number. ${ }^{*}$ pT0 refers to pathological stage post neoadjuvant therapy (NAT).

Table 3. Clinico-pathological data of 695 patients with HER2-positive breast cancer based on AR expression.

\begin{tabular}{|c|c|c|c|c|}
\hline & & $\begin{array}{c}\mathrm{AR}- \\
(n=58)\end{array}$ & $\begin{array}{c}\mathrm{AR}+ \\
(n=637)\end{array}$ & $p$-Value \\
\hline \multicolumn{2}{|c|}{ Median (IQR) age, years } & $55(44-68)$ & $59(49-68)$ & 0.045 \\
\hline \multirow[t]{2}{*}{ Age (year), $n(\%)$} & $<50$ & $23(39.7)$ & $161(25.3)$ & 0.020 \\
\hline & $\geq 50$ & $35(60.3)$ & $476(74.7)$ & \\
\hline \multirow{2}{*}{ Site, $n(\%)$} & Left & $32(55.2)$ & $364(57.4)$ & \multirow{2}{*}{0.740} \\
\hline & Right & $26(44.8)$ & $270(42.3)$ & \\
\hline \multirow{6}{*}{$\begin{array}{l}\text { Histologic type, } \\
n(\%)\end{array}$} & CDI & $52(89.7)$ & $538(84.6)$ & \multirow{6}{*}{0.770} \\
\hline & CLI & $1(1.7)$ & $29(4.6)$ & \\
\hline & CDI + CLI & $0(0.0)$ & $13(2.0)$ & \\
\hline & Apocrine & $3(5.2)$ & $36(5.7)$ & \\
\hline & Micropapillary + papillary & $1(1.7)$ & $14(2.2)$ & \\
\hline & Mucinous & $1(1.7)$ & $6(0.9)$ & \\
\hline \multirow{2}{*}{$\begin{array}{l}\text { Histologic grade, } \\
n(\%)\end{array}$} & G2 & $3(5.2)$ & $109(17.3)$ & \multirow{2}{*}{0.020} \\
\hline & G3 & $55(94.8)$ & $520(82.7)$ & \\
\hline \multirow{5}{*}{$\begin{array}{l}\text { Pathological tumor } \\
\text { size, } n(\%)\end{array}$} & pT0* & $4(7.6)$ & $49(8.6)$ & \multirow{5}{*}{0.020} \\
\hline & pT1 & $11(20.8)$ & $192(33.7)$ & \\
\hline & pT2 & $25(47.2)$ & $263(46.2)$ & \\
\hline & pT3 & $7(13.2)$ & $20(3.5)$ & \\
\hline & pT4 & $6(11.3)$ & $45(7.9)$ & \\
\hline \multirow{4}{*}{$\begin{array}{l}\text { Pathological lymph } \\
\text { node status, } n(\%)\end{array}$} & $\mathrm{pN} 0$ & $20(46.5)$ & $301(56.7)$ & \multirow{4}{*}{0.430} \\
\hline & $\mathrm{pN} 1$ & $14(32.6)$ & $120(22.6)$ & \\
\hline & $\mathrm{pN} 2$ & $5(11.6)$ & $68(12.8)$ & \\
\hline & $\mathrm{pN} 3$ & $4(9.3)$ & $42(7.9)$ & \\
\hline
\end{tabular}


Table 3. Cont.

\begin{tabular}{|c|c|c|c|c|}
\hline & & $\begin{array}{c}\mathrm{AR}- \\
(n=58)\end{array}$ & $\underset{(n=637)}{\mathrm{AR}+}$ & $p$-Value \\
\hline \multirow{3}{*}{$\begin{array}{l}\text { Lymph node ratio, } \\
n(\%)\end{array}$} & $\leq 0.20$ & $26(65.0)$ & $374(72.9)$ & \multirow{3}{*}{0.470} \\
\hline & $0.21-0.65$ & $10(25.0)$ & $96(18.7)$ & \\
\hline & $>0.65$ & $4(10.0)$ & $43(8.4)$ & \\
\hline \multirow{4}{*}{$\begin{array}{c}\text { Prognostic stage, } \\
n(\%)\end{array}$} & I & $8(16.3)$ & $241(42.8)$ & \multirow{4}{*}{0.001} \\
\hline & II & $22(44.9)$ & $152(27.0)$ & \\
\hline & III & $13(26.5)$ & $122(21.7)$ & \\
\hline & IV & $6(12.2)$ & $48(8.5)$ & \\
\hline \multicolumn{2}{|c|}{ Metastasis, $n(\%)$} & $6(10.3)$ & $48(7.5)$ & 0.440 \\
\hline \multirow{2}{*}{$\begin{array}{l}\text { Proliferation index } \\
(\mathrm{Ki}-67), n(\%)\end{array}$} & $<14 \%$ & $0(0.0)$ & $9(1.4)$ & \multirow{2}{*}{1.000} \\
\hline & $\geq 14 \%$ & $58(100.0)$ & $627(98.6)$ & \\
\hline \multirow{2}{*}{ ER expression, $n(\%)$} & $<10 \%$ & $40(69.0)$ & $241(37.8)$ & \multirow{2}{*}{$<0.0001$} \\
\hline & $\geq 10 \%$ & $18(31.0)$ & $396(62.2)$ & \\
\hline \multirow{2}{*}{ PR expression, $n(\%)$} & $<10 \%$ & $51(87.9)$ & $404(63.4)$ & \multirow{2}{*}{$<0.0001$} \\
\hline & $\geq 10 \%$ & $7(12.1)$ & $233(36.6)$ & \\
\hline \multirow{2}{*}{ AR expression, $n(\%)$} & $<10 \%$ & $57(98.3)$ & $2(0.3)$ & \multirow{2}{*}{$<0.0001$} \\
\hline & $\geq 10 \%$ & $1(1.7)$ & $635(99.7)$ & \\
\hline \multirow[t]{2}{*}{$\begin{array}{c}\text { HER2 expression, } \\
n(\%)\end{array}$} & $2+$ & $11(19.0)$ & $148(23.2)$ & \multirow[t]{2}{*}{0.680} \\
\hline & $3+$ & $48(81.0)$ & $489(76.8)$ & \\
\hline \multicolumn{2}{|c|}{ Mortality, $n(\%)$} & $12(20.7)$ & $87(13.7)$ & 0.140 \\
\hline
\end{tabular}

IQR: Interquartile range, $n$ : Number. ${ }^{*}$ pT0 refers to pathological stage post NAT.

Luminal and non-luminal HER2 $+\mathrm{AR}+\mathrm{BC}$ showed a higher age at diagnosis compared to luminal and non-luminal HER2+ AR- BCs. HER2+ AR - and HER2+ AR+ subtypes showed less frequently a lobular histotype compared to luminal HER2+ ARand AR+ subtypes. Mixed histological type was most represented in LB HER2+ subtypes expressing AR; HER2+ AR+ subtype was mainly associated with apocrine phenotype, while HER2+ AR - showed mucinous phenotype more frequently compared to the other subtypes. Histological grade G2 was mainly found in LB HER2+ AR+ (25.5\%). HER2+ subtypes without AR expression showed the highest pT3 and pT4 rates, accounting for $13.5 \%$ and $10.8 \%$ (HER2+ AR-), and $12.5 \%$ and $12.5 \%$ (LB HER2+ AR-), respectively.

Prognostic stage I was found more frequently in LB HER2+ AR+ $(55.5 \%)$, and less frequently in HER2+ AR - (6.3\%). On the other hand, stage III was mainly found in HER2+, both $\mathrm{AR}$ - and AR+ (28.1\% vs. 28.8). HER2+ AR - and HER2+ AR+ subtypes showed a significantly higher frequency of HER2 expression with score $3+$ (Table 4).

Table 4. Clinico-pathological data of 695 patients with HER2-positive breast cancer based on IHC subtypes.

\begin{tabular}{|c|c|c|c|c|c|c|}
\hline & & $\begin{array}{c}\text { LB HER } 2+\mathrm{AR}- \\
\quad(n=18)\end{array}$ & $\begin{array}{l}\text { LB HER2+ AR+ } \\
(n=396)\end{array}$ & $\begin{array}{l}\mathrm{HER} 2+\mathrm{AR}- \\
\quad(n=40)\end{array}$ & $\begin{array}{l}\text { HER2+ AR+ } \\
\quad(n=241)\end{array}$ & $p$-Value \\
\hline \multicolumn{2}{|c|}{ Median (IQR) age, years } & $53.5(41-62)$ & $59(48-69)$ & $55(45-70)$ & $59(50-68)$ & 0.160 \\
\hline \multirow{2}{*}{ Age (year), $n(\%)$} & $<50$ & $8(44.4)$ & $110(27.8)$ & $15(37.5)$ & $51(21.2)$ & \multirow{2}{*}{0.030} \\
\hline & $\geq 50$ & $10(55.6)$ & $286(72.2)$ & $25(62.5)$ & $190(78.8)$ & \\
\hline \multirow{2}{*}{ Site, $n(\%)$} & Left & $11(61.1)$ & $230(58.4)$ & $21(52.5)$ & $134(55.8)$ & \multirow{2}{*}{0.870} \\
\hline & Right & $7(38.9)$ & $164(41.6)$ & $19(47.5)$ & $106(44.2)$ & \\
\hline
\end{tabular}


Table 4. Cont.

\begin{tabular}{|c|c|c|c|c|c|c|}
\hline & & $\begin{array}{c}\text { LB HER2+ AR - } \\
(n=18)\end{array}$ & $\begin{array}{l}\text { LB HER2+ AR+ } \\
(n=396)\end{array}$ & $\begin{array}{c}\text { HER } 2+\text { AR - } \\
\quad(n=40)\end{array}$ & $\begin{array}{c}\text { HER2+ AR+ } \\
\quad(n=241)\end{array}$ & $p$-Value \\
\hline \multirow{6}{*}{$\begin{array}{l}\text { Histologic type, } \\
n(\%)\end{array}$} & CDI & $16(88.9)$ & $338(85.4)$ & $36(90.0)$ & $200(83.3)$ & \multirow{6}{*}{$<0.0001$} \\
\hline & CLI & $1(5.6)$ & $25(6.3)$ & $0(0.0)$ & $4(1.7)$ & \\
\hline & CDI + CLI & $0(0.0)$ & $10(2.5)$ & $0(0.0)$ & $3(1.3)$ & \\
\hline & Apocrine & $1(5.6)$ & $8(2.0)$ & $2(5.0)$ & 28 (11.7) & \\
\hline & $\begin{array}{l}\text { Micropapillary } \\
\text { + papillary }\end{array}$ & $0(0.0)$ & $11(2.7)$ & $1(2.5)$ & $3(1.3)$ & \\
\hline & Mucinous & $0(0.0)$ & $4(1.0)$ & $1(2.5)$ & $2(0.8)$ & \\
\hline \multirow{2}{*}{$\begin{array}{l}\text { Histologic grade, } \\
n(\%)\end{array}$} & G2 & $1(5.6)$ & $100(25.5)$ & $2(5.0)$ & $9(3.8)$ & \multirow{2}{*}{$<0.0001$} \\
\hline & G3 & $17(94.4)$ & $292(74.5)$ & $38(95.0)$ & $228(96.2)$ & \\
\hline \multirow{5}{*}{$\begin{array}{l}\text { Pathological tumor } \\
\text { size, } n(\%)\end{array}$} & pT0* & $2(12.5)$ & $20(5.7)$ & $2(5.4)$ & 29 (13.2) & \multirow{5}{*}{0.007} \\
\hline & pT1 & $4(25.0)$ & $127(36.3)$ & 7 (18.9) & 65 (29.6) & \\
\hline & pT2 & $6(37.5)$ & $162(46.3)$ & $19(51.4)$ & $101(45.9)$ & \\
\hline & pT3 & $2(12.5)$ & $15(4.3)$ & $5(13.5)$ & $5(2.3)$ & \\
\hline & pT4 & $2(12.5)$ & $26(7.4)$ & $4(10.8)$ & $20(9.1)$ & \\
\hline \multirow{4}{*}{$\begin{array}{l}\text { Pathological lymph } \\
\text { node status, } n(\%)\end{array}$} & pN0 & $6(40.0)$ & $182(55.8)$ & $14(50.0)$ & $119(58.1)$ & \multirow{4}{*}{0.570} \\
\hline & $\mathrm{pN} 1$ & $4(26.7)$ & 75 (23.0) & $10(35.7)$ & $45(22.0)$ & \\
\hline & $\mathrm{pN} 2$ & $3(20.0)$ & $46(14.1)$ & $2(7.1)$ & $22(10.7)$ & \\
\hline & $\mathrm{pN} 3$ & $2(13.3)$ & $23(7.1)$ & $2(7.1)$ & $19(9.3)$ & \\
\hline \multirow{3}{*}{$\begin{array}{l}\text { Lymph node ratio, } \\
n(\%)\end{array}$} & $\leq 0.20$ & $7(50.0)$ & $227(70.9)$ & $19(73.1)$ & $147(76.2)$ & \multirow{3}{*}{0.300} \\
\hline & $0.21-0.65$ & $6(42.9)$ & $64(20.0)$ & $4(15.4)$ & 32 (16.6) & \\
\hline & $>0.65$ & $1(7.1)$ & $29(9.1)$ & $3(11.5)$ & $14(7.3)$ & \\
\hline \multirow{4}{*}{$\begin{array}{c}\text { Prognostic stage, } \\
n(\%)\end{array}$} & I & $6(35.3)$ & $193(55.5)$ & $2(6.3)$ & $48(22.3)$ & \multirow{4}{*}{$<0.0001$} \\
\hline & II & $4(23.5)$ & 64 (18.4) & $18(56.3)$ & 88 (40.9) & \\
\hline & III & $4(23.5)$ & $60(17.2)$ & $9(28.1)$ & $62(28.8)$ & \\
\hline & IV & 3 (17.7) & $31(8.9)$ & $3(9.4)$ & $17(7.9)$ & \\
\hline Metastasi & $n(\%)$ & 3 (16.7) & $32(8.1)$ & $3(7.5)$ & $16(6.6)$ & 0.400 \\
\hline \multirow{4}{*}{$\begin{array}{l}\text { Proliferation index } \\
\quad(\mathrm{Ki}-67), n(\%)\end{array}$} & $<20 \%$ & $1(5.6)$ & $30(7.6)$ & $0(0.0)$ & $11(5.0)$ & \multirow{2}{*}{0.180} \\
\hline & $\geq 20 \%$ & 17 (94.4) & $366(92.4)$ & $40(100.0)$ & $229(95.0)$ & \\
\hline & $<14 \%$ & $0(0.0)$ & $6(1.5)$ & $0(0.0)$ & $3(1.2)$ & \multirow{2}{*}{1.000} \\
\hline & $\geq 14 \%$ & $18(100.0)$ & 390 (98.5) & $40(100.0)$ & $238(98.8)$ & \\
\hline \multirow{2}{*}{$\begin{array}{c}\text { ER expression, } \\
n(\%)\end{array}$} & $<10 \%$ & $0(0.0)$ & $0(0.0)$ & $40(100.0)$ & $241(100.0)$ & \multirow{2}{*}{$<0.0001$} \\
\hline & $\geq 10 \%$ & $18(100.0)$ & $396(100.0)$ & $0(0.0)$ & $0(0.0)$ & \\
\hline \multirow{2}{*}{$\begin{array}{l}\text { PR expression, } \\
n(\%)\end{array}$} & $<10 \%$ & $11(61.1)$ & $166(41.9)$ & $40(100.0)$ & $238(98.8)$ & \multirow{2}{*}{$<0.0001$} \\
\hline & $\geq 10 \%$ & $7(38.9)$ & $230(58.1)$ & $0(0.0)$ & $3(1.2)$ & \\
\hline \multirow{2}{*}{$\begin{array}{l}\text { AR expression, } \\
n(\%)\end{array}$} & $<10 \%$ & $17(94.4)$ & $0(0.0)$ & $40(100.0)$ & $2(0.8)$ & \multirow{2}{*}{$<0.0001$} \\
\hline & $\geq 10 \%$ & $1(5.6)$ & $396(100.0)$ & $0(0.0)$ & $239(99.2)$ & \\
\hline \multirow{2}{*}{$\begin{array}{c}\text { HER2 expression, } \\
n(\%)\end{array}$} & $2+$ & $6(33.3)$ & $126(31.8)$ & $5(12.5)$ & $229.1)$ & \multirow{2}{*}{$<0.0001$} \\
\hline & $3+$ & $12(66.7)$ & $270(68.1)$ & $35(87.5)$ & $219(90.9)$ & \\
\hline \multicolumn{2}{|c|}{ Mortality, $n(\%)$} & $3(16.7)$ & $45(11.4)$ & $9(22.5)$ & $42(17.4)$ & 0.060 \\
\hline
\end{tabular}


Prognostic Indicators according to HER2+ BC Subtypes

Factors influencing survival in the univariate analysis were: LB HER2+ AR+ subtype ( $p$ : 0.010); age at diagnosis $(p<0.0001)$; histological type $(p: 0.006)$; histological grade $(p$ : $0.003)$; tumor size $(p<0.0001)$; lymph node status and ratio $(p<0.0001$ for both); prognostic stage ( $p<0.0001)$; metastasis $(p<0.0001)$; Ki-67 expression ( $p: 0.002)$; ER, PR, and AR expression ( $p: 0.008, p: 0.010$, and $p: 0.008$, respectively) (Table 5). Multivariate analysis showed that histological type and AR expression are independent prognostic factors (OR: 0.36, 95\% CI 0.18-0.75, p: 0.006; OR: 0.99, 95\% CI 0.98-1.00, $p: 0.050$, respectively) (Table 5).

Table 5. Univariate and multivariate analysis for overall survival in HER2-positive breast cancer.

\begin{tabular}{|c|c|c|c|c|c|}
\hline & & \multicolumn{2}{|c|}{ Univariate Analysis } & \multicolumn{2}{|c|}{ Multivariate Analysis } \\
\hline & & OR $(95 \% \mathrm{CI})$ & $p$-Value & OR $(95 \%$ CI) & $p$-Value \\
\hline \multicolumn{2}{|c|}{ LB HER2 + AR - } & $1.21(0.34-4.26)$ & 0.770 & - & - \\
\hline \multicolumn{2}{|c|}{ LB HER2 + AR+ } & $0.58(0.38-0.89)$ & 0.010 & $4.17(0.29-59.39)$ & 0.290 \\
\hline \multicolumn{2}{|c|}{$\mathrm{HER} 2+\mathrm{AR}-$} & $1.82(0.84-3.96)$ & 0.130 & - & - \\
\hline \multicolumn{2}{|c|}{$\mathrm{HER} 2+\mathrm{AR}+$} & $1.47(0.95-2.27)$ & 0.080 & - & - \\
\hline \multicolumn{2}{|c|}{ Age, years } & $1.03(1.01-1.05)$ & $<0.0001$ & $1.02(0.99-1.04)$ & 0.200 \\
\hline \multicolumn{2}{|c|}{ Age $\geq 50$ years } & $1.50(0.89-2.53)$ & 0.130 & - & - \\
\hline \multicolumn{2}{|c|}{$\begin{array}{l}\text { Histologic type, } \\
\text { CDI VS. others }\end{array}$} & $0.49(0.29-0.81)$ & 0.006 & $0.36(0.18-0.75)$ & 0.006 \\
\hline \multirow{2}{*}{\multicolumn{2}{|c|}{ Histologic grade G3 VS. G2 }} & $4.08(1.62-10.27)$ & 0.003 & $2.21(0.68-7.18)$ & 0.190 \\
\hline & & OR $(95 \% \mathrm{CI})$ & $p$-Value & OR $(95 \%$ CI) & $p$-Value \\
\hline \multicolumn{2}{|c|}{ Tumor size, from pT0 to pT4 } & $2.49(1.96-3.17)$ & $<0.0001$ & - & - \\
\hline \multirow{5}{*}{$\begin{array}{l}\text { Pathological } \\
\text { tumor size }\end{array}$} & $\mathrm{pT} 0 *$ & Ref. & - & - & \\
\hline & pT1 & $1.05(0.22-5.08)$ & 0.960 & $1.31(0.24-7.11)$ & 0.750 \\
\hline & pT2 & 3.99 (0.93-17.07) & 0.060 & $2.51(0.53-11.87)$ & 0.240 \\
\hline & pT3 & $4.44(0.76-25.97)$ & 0.100 & $2.56(0.34-19.48)$ & 0.360 \\
\hline & pT4 & $\begin{array}{c}23.61 \\
(5.20-107.30)\end{array}$ & $<0.0001$ & $5.25(0.87-31.47)$ & 0.070 \\
\hline \multicolumn{2}{|c|}{$\begin{array}{l}\text { Pathological lymph node } \\
\text { status, from pN0 to pN3 }\end{array}$} & $2.12(1.65-2.71)$ & $<0.0001$ & - & - \\
\hline \multirow{4}{*}{$\begin{array}{l}\text { Pathological } \\
\text { lymph node } \\
\text { status }\end{array}$} & pN0 & Ref. & Ref. & - & - \\
\hline & $\mathrm{pN} 1$ & $2.40(1.15-5.01)$ & 0.020 & $1.20(0.47-3.11)$ & 0.700 \\
\hline & $\mathrm{pN} 2$ & $4.13(1.89-9.03)$ & $<0.0001$ & $2.11(0.49-9.15)$ & 0.320 \\
\hline & $\mathrm{pN} 3$ & $10.17(4.62-22.36)$ & $<0.0001$ & $3.17(0.57-17.69)$ & 0.190 \\
\hline \multicolumn{2}{|c|}{ Lymph node ratio } & 8.47 (3.76-19.10) & $<0.0001$ & - & - \\
\hline \multirow{3}{*}{$\begin{array}{l}\text { Lymph node } \\
\text { ratio }\end{array}$} & $\leq 0.20$ & Ref. & Ref. & - & - \\
\hline & $0.21-0.65$ & $2.67(1.37-5.20)$ & 0.004 & $1.06(0.35-3.21)$ & 0.910 \\
\hline & $>0.65$ & $6.36(3.02-13.40)$ & $<0.0001$ & $1.60(0.45-5.88)$ & 0.480 \\
\hline \multicolumn{2}{|c|}{ Prognostic stage, from I to IV } & $2.89(2.22-3.76)$ & $<0.0001$ & - & - \\
\hline \multirow{4}{*}{$\begin{array}{l}\text { Prognostic } \\
\text { stage }\end{array}$} & I & Ref. & Ref. & - & - \\
\hline & II & $3.26(1.38-7.74)$ & 0.007 & $1.43(0.47-4.42)$ & 0.530 \\
\hline & III & $8.61(3.82-19.40)$ & $<0.0001$ & $1.96(0.45-8.52)$ & 0.370 \\
\hline & IV & $\begin{array}{c}25.97 \\
(10.72-62.89)\end{array}$ & $<0.0001$ & $1.79(0.23-14.14)$ & 0.580 \\
\hline
\end{tabular}


Table 5. Cont.

\begin{tabular}{ccccc}
\hline & \multicolumn{2}{c}{ Univariate Analysis } & \multicolumn{2}{c}{ Multivariate Analysis } \\
\hline Metastasis & $7.23(4.02-12.99)$ & $<0.0001$ & - & - \\
\hline Proliferation index, \% & $1.02(1.01-1.03)$ & 0.002 & $1.02(0.99-1.04)$ & 0.150 \\
\hline $\begin{array}{c}\text { Proliferation index (Ki-67) } \\
\geq 14 \%\end{array}$ & - & - & - & - \\
\hline ER expression, \% & $0.99(0.99-1.00)$ & 0.008 & - & - \\
\hline ER expression $\geq 10 \%$ & $0.59(0.39-0.91)$ & 0.020 & $0.17(0.02-2.42)$ & 0.190 \\
\hline PR expression, \% & $0.99(0.99-1.00)$ & 0.070 & - & - \\
\hline PR expression $\geq 10 \%$ & $0.53(0.32-0.87)$ & 0.010 & $1.28(0.47-3.44)$ & 0.630 \\
\hline AR expression, \% & $0.99(0.98-0.99)$ & 0.008 & $0.99(0.98-1.00)$ & 0.050 \\
\hline AR expression $\geq 10 \%$ & $0.62(0.32-1.22)$ & 0.170 & - & - \\
\hline HER2, $\%$ & $1.00(0.99-1.01)$ & 0.480 & - & - \\
\hline HER2 expression 3 + VS. 2+ & $1.20(0.71-2.03)$ & 0.490 & - & - \\
\hline *pT0 refers to pathological stage post NAT. & & &
\end{tabular}

The Kaplan-Meier curve for OS showed no differences among BC HER2+ subtypes (Figure 2A). However, patients with G3 tumors had a worse survival ( $p$ : 0.004; Figure 2D). The best survival rate was found for those at stage I ( $p<0.0001$; Figure $2 \mathrm{C})$. A significant OS reduction was associated with increasing levels of lymph node ratio $(p<0.0001$; Figure $2 \mathrm{~B})$ and PR expression < 10\% ( $p$ : 0.040; Figure 2E).

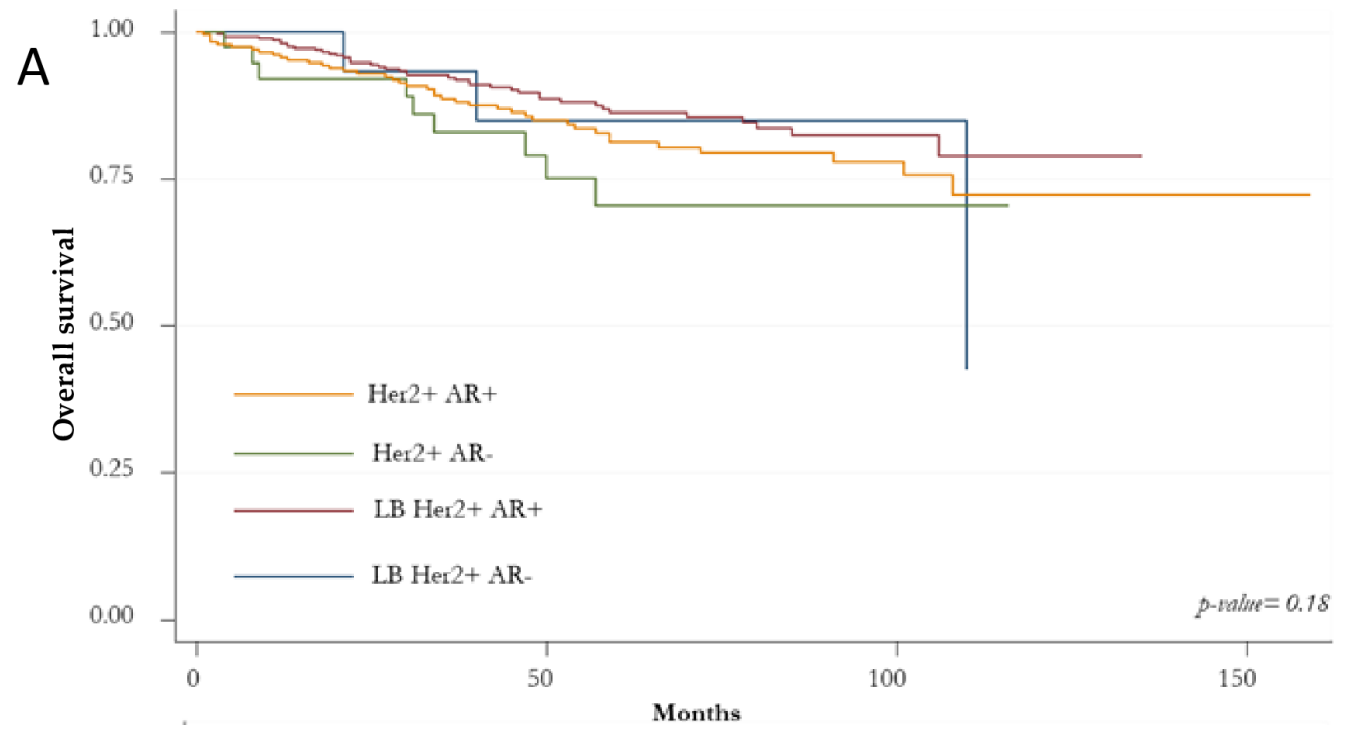

Figure 2. Cont. 

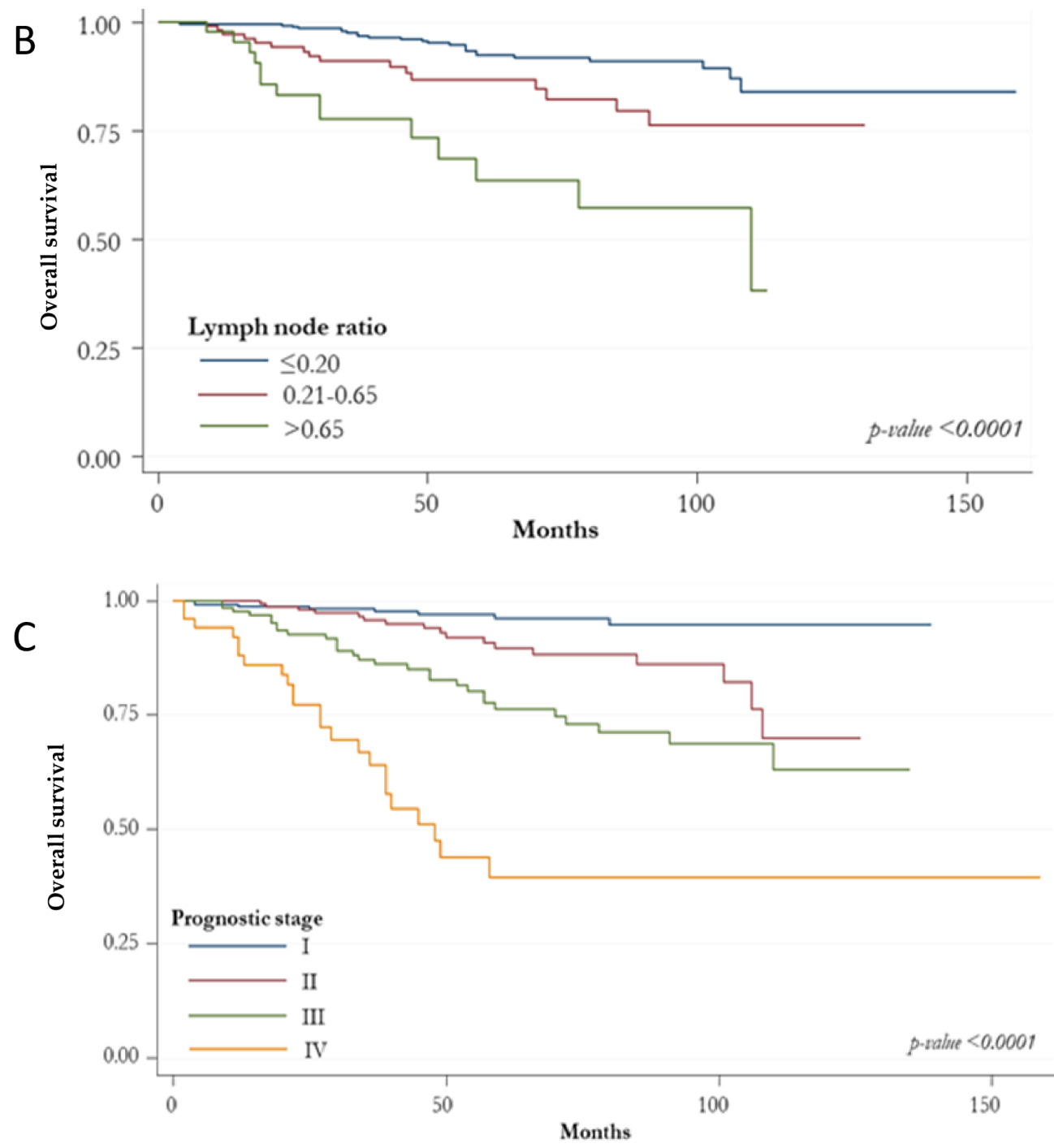

D

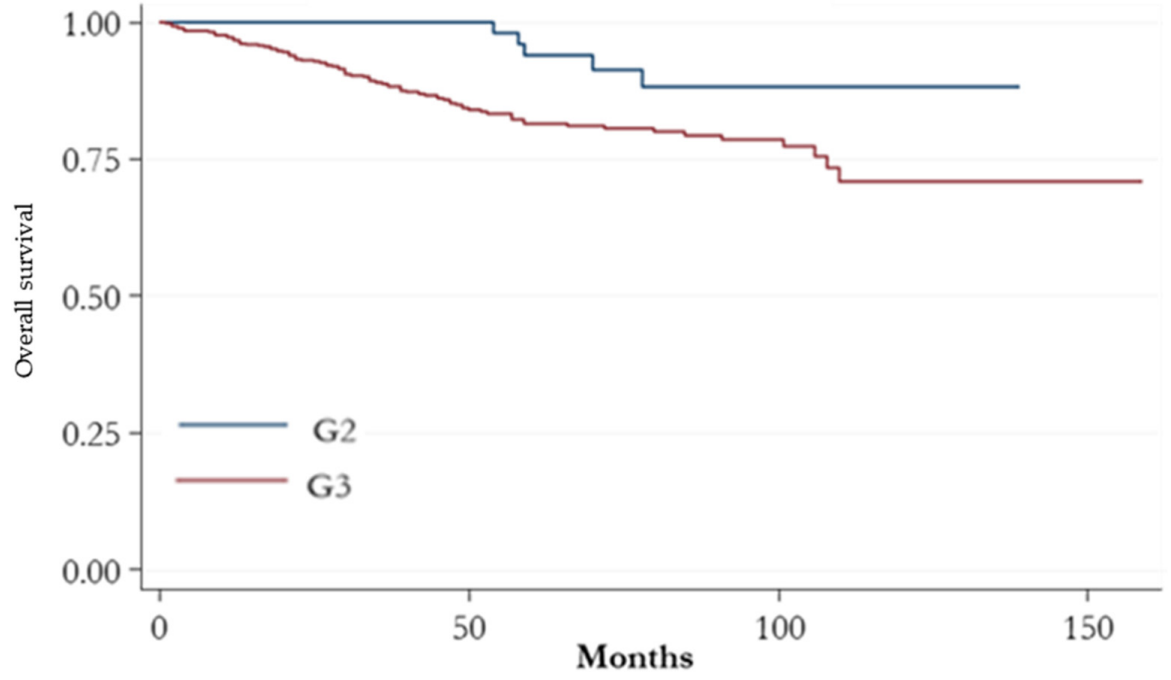

Figure 2. Cont. 


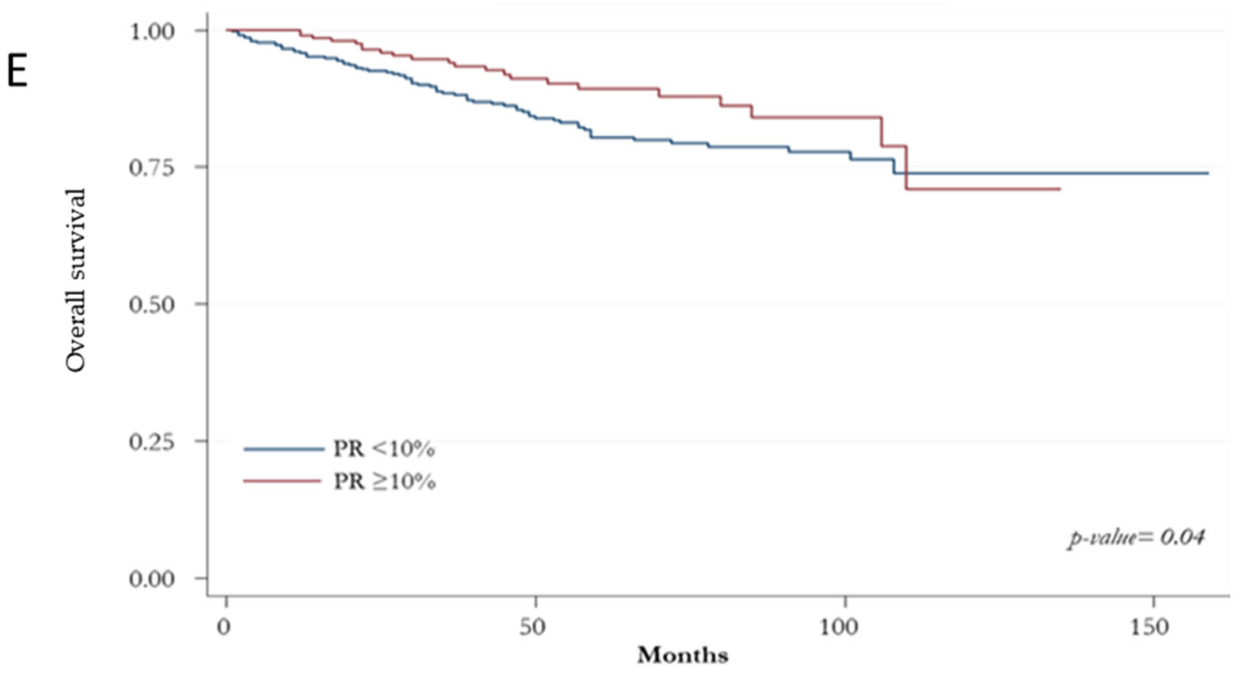

Figure 2. Kaplan-Meir curves for overall survival. (A) overall survival according to HER2-positive subtypes based on the state of the hormonal receptors (ER, PR and AR); (B) overall survival according to histologic grade; (C) overall survival according to prognostic stage; (D) overall survival according to lymph node ratio; (E) overall survival according to absence/presence of immunostaining for PR.

\section{Discussion}

BC HER2+ is an aggressive subtype including heterogeneous tumors with variable prognosis and treatment response to HER2-targeted therapies. On this matter, Staaf et al. identified three genetic BC HER2+ subtypes showing distinct clinical outcomes according to molecular profiling analysis [39]. The same group of researchers strengthened the molecular and biological complexity of BC HER2+ by showing the presence of high-level amplifications at multiple sites that also involved the HER2-amplicon at 17q12-q21 [39].

$\mathrm{AR}$ is a steroid hormone receptor frequently expressed in BC, including the ERnegative subtypes for which it could represent a complementary target for therapy, though the clinical significance and functional role of AR has not been outlined in BC yet $[40,41]$. The AR signaling pathway differs according to molecular breast cancer subtypes. It is known that in non-luminal HER2+ AR+ BCs, AR starts the WNT/ $\beta$-catenin activation, stimulating HER3 gene transcription, subsequently the heterodimers between HER3 and HER2 support cell proliferation [42]. Nevertheless, the AR signaling pathway in luminal HER2 + AR+ BCs is unclear, and the differences between AR signaling pathways in HER2+ BCs subtypes are not fully understood.

AR expression is higher than ER and PR in BC $[16,43]$. We found a high prevalence (91.5\%) of AR expression in BC HER2+, with minimal differences between luminal and non-luminal tumors; the absence or low level of AR expression being detected in a minority of tumors. These results are consistent with previous reports, which did not show significant differences between HER2-positive/ER-positive and HER2-positive/ER-negative tumors $[29,34,44-46]$. The higher percentage of AR expression in our cohort could depend on the definition of the AR expression, or on methods such as the use of the complete core section, population, and cut-off value.

Furthermore, our results showed that AR+ tumors are associated with older age at diagnosis and favorable clinical and pathological features, as well as lower histologic grade (G2), pT1 and prognostic stage (I), suggesting a prognostic value of AR in BC HER2+ subtypes. These findings are supported by previous studies showing the association of AR expression with lower grade, smaller size, more frequently tubule formation, and less pleomorphism and mitotic counts, although they evaluated ER-negative and ER-positive subgroups [17,47]. Besides, AR positivity was associated with high ER and PR expression in our cohort. Recently, Cruz-Tapias et al. evaluated the association of AR gene expression in accordance with intrinsic BC subtypes by meta-analysis of extensive microarray transcriptomic datasets. AR overexpression was prevalently observed in patients affected 
by less aggressive intrinsic molecular subtypes expressing either ER or PR and having a lower histological grade, such as Luminal A and B compared to Basal-like subtype. High AR mRNA levels can be defined as a prognostic biomarker for the detection of the less aggressive BC subtypes [48]. In the current study, the distinction of BC HER2+ in luminal and non-luminal showed that the lobular histotype is prevalent in luminal AR+ and ARtumors compared to non-luminal subtypes. The apocrine phenotype can be found in non-luminal HER2 + AR+ tumors, whereas AR negativity increases in the mucinous phenotype in the non-luminal subtype. These data consent to distinguish a different phenotype between luminal and non-luminal BC HER2 + AR+ subtypes, adding more detail to Park's results [17].

Moreover, the luminal BC HER2+ AR+ was associated with lower histological grade (G2) and lower tumor size, higher PR expression, and a lower HER2 intensity of expression (2+). Also, non-luminal tumors AR+ showed smaller size and prognostic stage (I) but higher grade (G3) and higher HER2 intensity of expression (3+). These results agree with the observations of other studies that have detected HER2 overexpression in G3-AR+ carcinomas $[16,29,49]$. These findings could suggest a different progression of luminal and non-luminal tumors both expressing AR, and how AR-related molecular mechanisms of BC HER2+ AR+ could differ depending on ER and PR expression.

Traditionally, the BC HER2+ subtype is distinguished in luminal HER2+ and nonluminal HER2+. In our study, AR positivity was detected in $91.5 \%$ of all tumors analyzed. Moreover, AR is expressed in $84.2 \%$ of BC HER2+, in the absence of other hormonal receptors, namely ER and PR. From a clinical point of view, tumors with AR expression show a slightly better clinical outcome. These findings suggest that the immunophenotype classification is not completely exhaustive; like TNBC [50], the presence of AR could confer a luminal phenotype. The concurrent presence with the estrogen receptor could make the androgen receptor a favorable prognostic marker also in the BC HER2+ subtype.

The role of androgens and AR might vary depending on cancer cell types and/or on the level of expression of other steroid hormone receptors. In ER-positive tumors, AR has an anti-proliferative effect by antagonizing ER, by binding to a subset of estrogen response elements (EREs); it can prevent the activation of target genes which mediate the stimulatory effects of 17-beta-estradiol on breast cancer cells [51,52]. Recently, Hickey et al. demonstrated that AR performs a tumor suppressor role in the ER-positive BC subtype, and the AR activation induces potent antitumor activity in multiple disease contexts, including resistance to endocrine therapy and CDK4/6 inhibitors. These data reinforce AR agonism as the optimal AR-directed treatment strategy, showing a rational therapeutic opportunity for this tumor subtype.

However, androgens may have a proliferative effect through AR in ER-AR+ tumor cells. In HER2+ tumors, AR triggers the WNT/ $\beta$-catenin pathway causing HER3 upregulation; through HER2/HER3 heterodimers, it can cause the activation of the PI3K/AKT pathway and may lead to cell proliferation through MYC $[53,54]$. Furthermore, in nonluminal BC HER2+, AR induces HER2 expression, which in turn leads to ERK activation, which requires HER2 and AR activity. These findings suggest that HER2 is an upstream connector between the AR and ERK signaling pathways. Another feature of this feedback loop is an ERK-mediated regulation of AR [55]. Previous findings should have potential clinical relevance; considering the He's study, the inhibition of AR with enzalutamide or shRNAs decreases the growth of HER2+ BC cells in vitro and in vivo, having a sensitivity similar to the trastuzumab. Interestingly, the inhibition of AR diminished the phosphorylation of HER2 and the activation of AKT and ERK without involving HER2 and HER3 protein expression levels. These findings indicate a new role of AR in HER2 signaling, and anti-AR target therapy may be beneficial in HER2+ BC patients that are unresponsive or that develop resistance to anti-HER2 therapies [56].

The prognostic role of AR in BC ER-negative and PR-negative has been poorly evaluated, in comparison with BC ER-positive PR-positive variants [57]. Our study, which was focused on luminal and non-luminal BC HER2+ showed that histological type and 
AR expression are good independent prognostic factors for OS in the HER2+ BC patients. Furthermore, it was found that a poorer OS in BC HER2-positive patients with higher histological grade (G3), higher level of lymph node ratio and PR expression $<10 \%$. Wang et al. demonstrated the association between BC HER2+ expressing AR and longer progressionfree survival, increased five years OS rate, and the efficacy of trastuzumab therapy [34]. Akashi et al. found that AR expression was associated with the significant effectiveness of neoadjuvant chemotherapy and prognosis in HER2+ tumors [46]. Our study does have some limitations, that are primarily focused on its retrospective strategy. Hence, some information on clinical follow-up data were not completely included in the medical records. Additionally, the analysis should be expanded to more patients in the coming years to consolidate and reproduce our results.

\section{Conclusions}

AR is frequently expressed in BC HER2+ subtypes. Our results showed that AR expression has a prognostic value in BC HER2+ subtypes, with better clinical outcomes. A better prognosis was highlighted in luminal HER2+ AR+ tumors subtype compared to the non-luminal HER2+ AR+ subtype, based on clinico-pathological data. AR expression should be assessed to evaluate the prognosis of BC HER2+ subtypes. Moreover, the understanding of the complex interactions between AR and the HER2 signaling pathway could pave the way to the use of AR as a therapeutic target in BC HER2+ subtypes.

Author Contributions: Conceptualization, M.R.D.M. and S.O.; methodology, P.C.-R., D.P., C.C., C.A., G.P., R.B. and C.M.; formal analysis, G.S., L.S., E.P. and M.R.M.; writing-original draft preparation, M.R.D.M.; writing-review and editing, P.C.-R., S.O., E.P. and G.S.; funding acquisition, M.R.D.M. All authors have read and agreed to the published version of the manuscript.

Funding: This work was partly supported by grants from Fondazione Banco di Sardegna, Italy, and "Fondo di Ateneo per la Ricerca 2020" from University of Sassari, Italy.

Institutional Review Board Statement: The study was conducted according to the guidelines of the Declaration of Helsinki and approved by the Independent Ethics Committee of the Azienda Ospedaliera Brotzu (AOB) of Cagliari, Italy (File number PG/2021/14264).

Informed Consent Statement: Informed consent statement for this study was not needed, according to the Italian legislation on the guidelines for observational studies (G.U. n. 76. 31-3-2008).

Data Availability Statement: Not applicable.

Conflicts of Interest: The authors declare no conflict of interest.

\section{References}

1. Sanges, F.; Floris, M.; Cossu-Rocca, P.; Muroni, M.R.; Pira, G.; Urru, S.A.M.; Barrocu, R.; Gallus, S.; Bosetti, C.; D'Incalci, M.; et al. Histologic subtyping affecting outcome of triple negative breast cancer: A large Sardinian population-based analysis. BMC Cancer 2020, 20, 49. [CrossRef] [PubMed]

2. Uva, P.; Cossu-Rocca, P.; Loi, F.; Pira, G.; Murgia, L.; Orrù, S.; Floris, M.; Muroni, M.R.; Sanges, F.; Carru, C.; et al. miRNA135b Contributes to Triple Negative Breast Cancer Molecular Heterogeneity: Different Expression Profile in Basal-like Versus non-Basal-like Phenotypes. Int. J. Med. Sci. 2018, 15, 536-548. [CrossRef]

3. Li, J.; Chen, Z.; Su, K.; Zeng, J. Clinicopathological classification and traditional prognostic indicators of breast cancer. Int. J. Clin. Exp. Pathol. 2015, 8, 8500-8505.

4. Boyle, P. Triple-negative breast cancer: Epidemiological considerations and recommendations. Ann. Oncol. Off. J. Eur. Soc. Med. Oncol. 2012, 23 (Suppl. 6), vi7-vi12. [CrossRef] [PubMed]

5. Rakha, E.A.; Allison, K.H.; Ellis, I.O.; Horii, R.; Masuda, S.; Penault-Llorca, F.; Tsuda, H.; Vincent-Salomon, A. Invasive breast carcinoma: General overview. In WHO Classification of Tumours, 5th ed.; WHO Classification of Tumours Editorial Board, Ed.; IARC Press: Lyon, France, 2019; pp. 82-101.

6. Wolff, A.C.; Hammond, M.E.H.; Hicks, D.G.; Dowsett, M.; McShane, L.M.; Allison, K.H.; Allred, D.C.; Bartlett, J.M.S.; Bilous, M.; Fitzgibbons, P.; et al. Recommendations for human epidermal growth factor receptor 2 testing in breast cancer: American Society of Clinical Oncology/College of American Pathologists clinical practice guideline update. J. Clin. Oncol. 2013, 31, 3997-4013. [CrossRef] 
7. Dittrich, A.; Gautrey, H.; Browell, D.; Tyson-Capper, A. The HER2 Signaling Network in Breast Cancer-Like a Spider in its Web. J. Mammary Gland Biol. Neoplasia 2014, 19, 253-270. [CrossRef] [PubMed]

8. Burstein, H.J.; Curigliano, G.; Loibl, S.; Dubsky, P.; Gnant, M.; Poortmans, P.; Colleoni, M.; Denkert, C.; Piccart-Gebhart, M.; Regan, M.; et al. Estimating the benefits of therapy for early-stage breast cancer: The St. Gallen International Consensus Guidelines for the primary therapy of early breast cancer 2019. Ann. Oncol. 2019, 30, 1541-1557. [CrossRef]

9. Wang, C.; Deng, S.; Chen, J.; Xu, X.; Hu, X.; Kong, D.; Liang, G.; Yuan, X.; Li, Y.; Wang, X. The Synergistic Effects of Pyrotinib Combined With Adriamycin on HER2-Positive Breast Cancer. Front. Oncol. 2021, 11, 1603. [CrossRef]

10. Vogel, C.; Cobleigh, M.A.; Tripathy, D.; Gutheil, J.C.; Harris, L.N.; Fehrenbacher, L.; Slamon, D.J.; Murphy, M.; Novotny, W.F.; Burchmore, M.; et al. First-line, single-agent Herceptin ${ }^{\circledR}$ (trastuzumab) in metastatic breast cancer: A preliminary report. Eur. J. Cancer 2001, 37, 25-29. [CrossRef]

11. Gijsen, M.; King, P.; Perera, T.; Parker, P.J.; Harris, A.L.; Larijani, B.; Kong, A. HER2 Phosphorylation Is Maintained by a PKB Negative Feedback Loop in Response to Anti-HER2 Herceptin in Breast Cancer. PLoS Biol. 2010, 8, e1000563. [CrossRef]

12. Scaltriti, M.; Rojo, F.; Ocaña, A.; Anido, J.; Guzman, M.; Cortes, J.; Di Cosimo, S.; Matias-Guiu, X.; Ramon y Cajal, S.; Arribas, J.; et al. Expression of p95HER2, a truncated form of the HER2 receptor, and response to anti-HER2 therapies in breast cancer. J. Natl. Cancer Inst. 2007, 99, 628-638. [CrossRef]

13. Nagata, Y.; Lan, K.H.; Zhou, X.; Tan, M.; Esteva, F.J.; Sahin, A.A.; Klos, K.S.; Li, P.; Monia, B.P.; Nguyen, N.T.; et al. PTEN activation contributes to tumor inhibition by trastuzumab, and loss of PTEN predicts trastuzumab resistance in patients. Cancer Cell 2004, 6, 117-127. [CrossRef] [PubMed]

14. Lu, Y.; Zi, X.; Pollak, M. Molecular mechanisms underlying IGF-I-induced attenuation of the growth-inhibitory activity of trastuzumab (Herceptin) on SKBR3 breast cancer cells. Int. J. Cancer 2004, 108, 334-341. [CrossRef] [PubMed]

15. Zhuang, G.; Brantley-Sieders, D.M.; Vaught, D.; Yu, J.; Xie, L.; Wells, S.; Jackson, D.; Muraoka-Cook, R.; Arteaga, C.; Chen, J. Elevation of receptor tyrosine kinase EphA2 mediates resistance to trastuzumab therapy. Cancer Res. 2010, 70, 299-308. [CrossRef] [PubMed]

16. Moinfar, F.; Okcu, M.; Tsybrovskyy, O.; Regitnig, P.; Lax, S.F.; Weybora, W.; Ratschek, M.; Tavassoli, F.A.; Denk, H. Androgen receptors frequently are expressed in breast carcinomas: Potential relevance to new therapeutic strategies. Cancer 2003, 98, 703-711. [CrossRef] [PubMed]

17. Park, S.; Koo, J.; Park, H.S.; Kim, J.H.; Choi, S.Y.; Lee, J.H.; Park, B.W.; Lee, K.S. Expression of androgen receptors in primary breast cancer. Ann. Oncol. 2010, 21, 488-492. [CrossRef]

18. Niemeier, L.A.; Dabbs, D.J.; Beriwal, S.; Striebel, J.M.; Bhargava, R. Androgen receptor in breast cancer: Expression in estrogen receptor-positive tumors and in estrogen receptor-negative tumors with apocrine differentiation. Mod. Pathol. 2010, 23, 205-212. [CrossRef] [PubMed]

19. Kensler, K.H.; Regan, M.M.; Heng, Y.J.; Baker, G.M.; Pyle, M.E.; Schnitt, S.J.; Hazra, A.; Kammler, R.; Thürlimann, B.; Colleoni, M.; et al. Prognostic and predictive value of androgen receptor expression in postmenopausal women with estrogen receptor-positive breast cancer: Results from the Breast International Group Trial 1-98. Breast Cancer Res. 2019, 21. [CrossRef]

20. Collins, L.C.; Cole, K.S.; Marotti, J.D.; Hu, R.; Schnitt, S.J.; Tamimi, R.M. Androgen receptor expression in breast cancer in relation to molecular phenotype: Results from the Nurses' Health Study. Mod. Pathol. 2011, 24, 924-931. [CrossRef]

21. Yu, Q.; Niu, Y.; Liu, N.; Zhang, J.Z.; Liu, T.J.; Zhang, R.J.; Wang, S.L.; Ding, X.M.; Xiao, X.Q. Expression of androgen receptor in breast cancer and its significance as a prognostic factor. Ann. Oncol. Off. J. Eur. Soc. Med. Oncol. 2011, 22, 1288-1294. [CrossRef]

22. Jiang, H.S.; Kuang, X.Y.; Sun, W.L.; Xu, Y.; Zheng, Y.Z.; Liu, Y.R.; Lang, G.T.; Qiao, F.; Hu, X.; Shao, Z.M. Androgen receptor expression predicts different clinical outcomes for breast cancer patients stratified by hormone receptor status. Oncotarget 2016, 7, 41285-41293. [CrossRef]

23. Bozovic-Spasojevic, I.; Zardavas, D.; Brohee, S.; Ameye, L.; Fumagalli, D.; Ades, F.; De Azambuja, E.; Bareche, Y.; Piccart, M.; Paesmans, M.; et al. The Prognostic Role of Androgen Receptor in Patients with Early-Stage Breast Cancer: A Meta-analysis of Clinical and Gene Expression Data. Clin. Cancer Res. 2017, 23, 2702-2712. [CrossRef]

24. De Amicis, F.; Thirugnansampanthan, J.; Cui, Y.; Selever, J.; Beyer, A.; Parra, I.; Weigel, N.L.; Herynk, M.H.; Tsimelzon, A.; Lewis, M.T.; et al. Androgen receptor overexpression induces tamoxifen resistance in human breast cancer cells. Breast Cancer Res. Treat. 2010, 121, 1-11. [CrossRef] [PubMed]

25. Rechoum, Y.; Rovito, D.; Iacopetta, D.; Barone, I.; Andò, S.; Weigel, N.L.; O’Malley, B.W.; Brown, P.H.; Fuqua, S.A.W. AR collaborates with ER $\alpha$ in aromatase inhibitor-resistant breast cancer. Breast Cancer Res. Treat. 2014, 147, 473-485. [CrossRef] [PubMed]

26. D'Amato, N.C.; Gordon, M.A.; Babbs, B.; Spoelstra, N.S.; Butterfield, K.T.C.; Torkko, K.C.; Phan, V.T.; Barton, V.N.; Rogers, T.J.; Sartorius, C.A.; et al. Cooperative Dynamics of AR and ER Activity in Breast Cancer. Mol. Cancer Res. 2016, 14, $1054-1067$. [CrossRef]

27. Gerratana, L.; Basile, D.; Buono, G.; De Placido, S.; Giuliano, M.; Minichillo, S.; Coinu, A.; Martorana, F.; De Santo, I.; Del Mastro, L.; et al. Androgen receptor in triple negative breast cancer: A potential target for the targetless subtype. Cancer Treat. Rev. 2018, 68, 102-110. [CrossRef] [PubMed]

28. Ricciardelli, C.; Bianco-Miotto, T.; Jindal, S.; Butler, L.M.; Leung, S.; McNeil, C.M.; O'Toole, S.A.; Ebrahimie, E.; Millar, E.K.A.; Sakko, A.J.; et al. The Magnitude of Androgen Receptor Positivity in Breast Cancer Is Critical for Reliable Prediction of Disease Outcome. Clin. Cancer Res. 2018, 24, 2328-2341. [CrossRef] [PubMed] 
29. Micello, D.; Marando, A.; Sahnane, N.; Riva, C.; Capella, C.; Sessa, F. Androgen receptor is frequently expressed in HER2-positive, ER/PR-negative breast cancers. Virchows Arch. 2010, 457, 467-476. [CrossRef] [PubMed]

30. Isola, J.J. Immunohistochemical demonstration of androgen receptor in breast cancer and its relationship to other prognostic factors. J. Pathol. 1993, 170, 31-35. [CrossRef] [PubMed]

31. Farmer, P.; Bonnefoi, H.; Becette, V.; Tubiana-Hulin, M.; Fumoleau, P.; Larsimont, D.; MacGrogan, G.; Bergh, J.; Cameron, D.; Goldstein, D.; et al. Identification of molecular apocrine breast tumours by microarray analysis. Oncogene 2005, 24, 4660-4671. [CrossRef]

32. Ni, M.; Chen, Y.; Lim, E.; Wimberly, H.; Bailey, S.T.; Imai, Y.; Rimm, D.L.; Shirley Liu, X.; Brown, M. Targeting Androgen Receptor in Estrogen Receptor-Negative Breast Cancer. Cancer Cell 2011, 20, 119-131. [CrossRef] [PubMed]

33. De Mattos Lima Lin, F.; Pincerato, K.M.; Bacchi, C.E.; Baracat, E.C.; Carvalho, F.M. Coordinated expression of oestrogen and androgen receptors in HER2-positive breast carcinomas: Impact on proliferative activity. J. Clin. Pathol. 2012, 65, 64-68. [CrossRef]

34. Wang, X.; Bi, X.; Huang, Z.; Huang, J.; Xia, W.; Shi, W.; Yuan, Z. The prognostic value of androgen receptor (AR) in HER2-enriched metastatic breast cancer. Endocr. Relat. Cancer 2020, 27, 199-208. [CrossRef]

35. Urru, S.A.M.; Gallus, S.; Bosetti, C.; Moi, T.; Medda, R.; Sollai, E.; Murgia, A.; Sanges, F.; Pira, G.; Manca, A.; et al. Clinical and pathological factors influencing survival in a large cohort of triple-negative breast cancer patients. BMC Cancer $2018,18$. [CrossRef]

36. Elston, C.W.; Ellis, I.O. Pathological prognostic factors in breast cancer. I. The value of histological grade in breast cancer: Experience from a large study with long-term follow-up. Histopathology 2002, 41, 154-161. [CrossRef] [PubMed]

37. Edge, S.B.; Compton, C.C. The american joint committee on cancer: The 7th edition of the AJCC cancer staging manual and the future of TNM. Ann. Surg. Oncol. 2010, 17, 1471-1474. [CrossRef]

38. Vinh-Hung, V.; Verkooijen, H.M.; Fioretta, G.; Neyroud-Caspar, I.; Rapiti, E.; Vlastos, G.; Deglise, C.; Usel, M.; Lutz, J.M.; Bouchardy, C. Lymph node ratio as an alternative to pN staging in node-positive breast cancer. J. Clin. Oncol. 2009, 27, 1062-1068. [CrossRef]

39. Staaf, J.; Jönsson, G.; Ringnér, M.; Vallon-Christersson, J.; Grabau, D.; Arason, A.; Gunnarsson, H.; Agnarsson, B.A.; Malmström, P.O.; Johannsson, O.T.; et al. High-resolution genomic and expression analyses of copy number alterations in HER2amplified breast cancer. Breast Cancer Res. 2010, 12,1-18. [CrossRef]

40. Nicolás Díaz-Chico, B.; Germán Rodríguez, F.; González, A.; Ramírez, R.; Bilbao, C.; Cabrera de León, A.; Aguirre Jaime, A.; Chirino, R.; Navarro, D.; Díaz-Chico, J.C. Androgens and androgen receptors in breast cancer. J. Steroid Biochem. Mol. Biol. 2007, 105, 1-15. [CrossRef]

41. Higgins, M.J.; Wolff, A.C. The androgen receptor in breast cancer: Learning from the past. Breast Cancer Res. Treat. 2010, 124, 619-621. [CrossRef]

42. Pietri, E.; Conteduca, V.; Andreis, D.; Massa, I.; Melegari, E.; Sarti, S.; Cecconetto, L.; Schirone, A.; Bravaccini, S.; Serra, P.; et al. Androgen receptor signaling pathways as a target for breast cancer treatment. Endocr. Relat. Cancer 2016, 23, R485-R498. [CrossRef] [PubMed]

43. Nahleh, Z. Androgen receptor as a target for the treatment of hormone receptor-negative breast cancer: An unchartered territory. Future Oncol. 2008, 4, 15-21. [CrossRef] [PubMed]

44. Zhao, L.; Niu, F.; Shen, H.; Liu, X.; Chen, L.; Niu, Y. Androgen receptor and metastasis-associated protein-1 are frequently expressed in estrogen receptor negative/HER2 positive breast cancer. Virchows Arch. 2016, 468, 687-696. [CrossRef] [PubMed]

45. Kucukzeybek, B.B.; Bayoglu, I.V.; Kucukzeybek, Y.; Yıldız, Y.; Oflazoglu, U.; Atahan, M.K.; Taskaynatan, H.; Alacacıolu, A.; Yigit, S.; Tarhan, M.O. Prognostic significance of androgen receptor expression in HER2-positive and triple-negative breast cancer. Pol. J. Pathol. 2018, 69, 157-168. [CrossRef]

46. Akashi, M.; Yamaguchi, R.; Kusano, H.; Ogasawara, S.; Abe, E.; Obara, H.; Yamaguchi, M.; Akiba, J.; Kakuma, T.; Tanaka, M.; et al. Androgen receptor expression is useful to predict the therapeutic effect in HER2-positive breast carcinoma. Breast Cancer Res. Treat. 2020, 184, 277-285. [CrossRef]

47. Rajković-Molek, K.; Mustać, E.; Avirović, M.; Georgev, P.; Demaria, M.; Aničić, J.; Ban, J.; Babarović, E. The expression of calpain-1 and androgen receptor in breast cancer and their correlation with clinicopathological characteristics: An immunohistochemical retrospective study. Pathol.-Res. Pract. 2020, 216, 153068. [CrossRef]

48. Cruz-Tapias, P.; Rubiano, W.; Rondón-Lagos, M.; Villegas, V.-E.; Rangel, N. Intrinsic Subtypes and Androgen Receptor Gene Expression in Primary Breast Cancer. A Meta-Analysis. Biology 2021, 10, 834. [CrossRef]

49. Liegl, B.; Horn, L.C.; Moinfar, F. Androgen receptors are frequently expressed in mammary and extramammary Paget's disease. Mod. Pathol. 2005, 18, 1283-1288. [CrossRef]

50. Barton, V.N.; D'Amato, N.C.; Gordon, M.A.; Christenson, J.L.; Elias, A.; Richer, J.K. Androgen Receptor Biology in Triple Negative Breast Cancer: A Case for Classification as AR+ or Quadruple Negative Disease. Horm. Cancer 2015, 6, 206-213. [CrossRef] [PubMed]

51. Peters, A.A.; Buchanan, G.; Ricciardelli, C.; Bianco-Miotto, T.; Centenera, M.M.; Harris, J.M.; Jindal, S.; Segara, D.; Jia, L.; Moore, N.L.; et al. Androgen Receptor Inhibits Estrogen Receptor- $\alpha$ Activity and Is Prognostic in Breast Cancer. Cancer Res. 2009, 69, 6131-6140. [CrossRef] [PubMed]

52. Rich, J.N. Cancer Stem Cells in Radiation Resistance. Cancer Res. 2007, 67, 8980-8984. [CrossRef] 
53. Turashvili, G.; Bouchal, J.; Burkadze, G.; Kolar, Z. Wnt signaling pathway in mammary gland development and carcinogenesis. Pathobiology 2006, 73, 213-223. [CrossRef]

54. Ni, M.; Chen, Y.; Fei, T.; Li, D.; Lim, E.; Liu, X.S.; Brown, M. Amplitude modulation of androgen signaling by c-MYC. Genes Dev. 2013, 27, 734-748. [CrossRef]

55. Chia, K.M.; Liu, J.; Francis, G.D.; Naderi, A. A feedback loop between androgen receptor and ERK signaling in estrogen receptor-negative breast cancer. Neoplasia 2011, 13, 154-166. [CrossRef]

56. He, L.; Du, Z.; Xiong, X.; Ma, H.; Zhu, Z.; Gao, H.; Cao, J.; Li, T.; Li, H.; Yang, K.; et al. Targeting Androgen Receptor in Treating HER2 Positive Breast Cancer. Sci. Rep. 2017, 7. [CrossRef]

57. Bryś, M.; Wójcik, M.; Romanowicz-Makowska, H.; Krajewska, W.M. Androgen receptor status in female breast cancer: RT-PCR and Western blot studies. J. Cancer Res. Clin. Oncol. 2002, 128, 85-90. [CrossRef] 